\title{
Stability Analysis for a Class of Networked/Embedded Control Systems: A Discrete-Time Approach
}

\author{
Hisaya Fujioka
}

\begin{abstract}
Motivated by the widespread use of networked and embedded control systems, an algorithm for stability analysis is proposed for sampled-data feedback control systems with uncertainly time-varying sampling intervals. The algorithm is based on the robustness of discrete-time systems against perturbation caused by the variation of sampling intervals. The validity of the algorithm is demonstrated by numerical examples.
\end{abstract}

Index Terms-networked control systems, sampled-data systems, quadratic stability, matrix exponential

\section{INTRODUCTION}

The sampled-data control theory (See [1] and references therein) has been well-developed in the last two decades, where the crucial properties is the periodicity of the closedloop systems which comes from the periodic sampling. It is reasonable to consider the periodic sampling in the conventional implementation of sampled-data systems. We, however, recently encounter applications where the periodic sampling is almost impossible. For example, resources for measurement and control are restricted in networked and/or embedded control systems (See [2], [3] and references therein), and hence the sampling operation results to be aperiodic and uncertainly time-varying. In view of the widespread use of networked and/or embedded control systems, it is theoretically and practically important to study the robustness of such systems against variation of sampling intervals. One can find pioneering work for the issue in the literature [4], [5], [6].

Recently the so-called input delay approach [7], [8] was proposed to treat the systems with aperiodic sampling, and a significant reduction of the conservatism is achieved. The basic idea of the approach is modeling the aperiodic sampleand-hold operations by a time-varying uncertain time delay at control input, and hence one can apply methodologies developed for delay systems to the aperiodic sampled-data systems. One can find applications of the input delay approach to several analysis and synthesis problems [7], [8], [9], [10], [11], [12]. Moreover this approach has inspired the discussion of the problem from the viewpoints of hybrid systems [13], [14] and robust control [15], [16]. It would be worth mentioning that most of existing results verify stability by showing the existence of a continuous-time Lyapunov function.

The purpose of this paper is to develop an algorithm to check the stability of the aperiodic sampled-data systems. We, however, take a different approach. Namely we will

H. Fujioka is with Graduate School of Informatics, Kyoto University, Kyoto 606-8501, Japan, fujioka@i.kyoto-u.ac.jp verify stability by showing the existence of a discrete-time quadratic Lyapunov function. This approach is also taken in [6] where a quadratic Lyapunov function is searched by a randomized algorithm. Their algorithm, however, checks the quadratic stability of a set of discrete-time systems related to finite number of prespecified sampling intervals between bounds of sampling intervals. In other words, the algorithm in [6] determines if a necessary condition (for a sufficient condition for the stability) holds or not, and hence cannot conclude the stability. In contrast we will derive an algorithm of checking the quadratic stability for all sampling intervals uncertainly varying between given lower and upper bounds, by exploiting the robustness against perturbation caused by the variation of sampling intervals based on the small-gain condition.

This paper is organized as follows: The problem is formulated in Section II. Section III provides a stability criteria and an algorithm to verify the stability based on the criteria. The validity of the algorithm is demonstrated in Section IV. We discuss some directions to reduce the conservatism in Section V.

\section{PRoblem Formulation}

Let the following state-space system be given

$$
\dot{x}(t)=A x(t)+B u(t)
$$

where $x$ and $u$ respectively denote the state and the input taking values in $\mathbb{R}^{n}$ and $\mathbb{R}^{m}$. $A$ and $B$ are matrices of compatible dimensions.

We consider the following scenario of the feedback control of (1):

- We can measure the state of (1) when $t=\tau_{k}(k=0$, $1, \ldots)$ where $\left\{\tau_{k}\right\}$ is an uncertain set of discrete time instances satisfying

$$
\tau_{0}=0
$$

and

$$
0<h_{\ell} \leq \tau_{k+1}-\tau_{k} \leq h_{u}<\infty
$$

for given $h_{\ell}$ and $h_{u}$.

- The control input $u$ is determined from the sampleddata $x\left(\tau_{k}\right)$ and a given feedback gain $F \in \mathbb{R}^{m \times n}$ by

$$
u(t)=F x\left(\tau_{k}\right), \quad{ }^{\forall} t \in\left[\tau_{k}, \tau_{k+1}\right) .
$$

The resultant feedback system composed of (1) and (4), denoted by $T$, is given by

$$
\dot{x}(t)=A x(t)+B F x\left(\tau_{k}\right), \quad{ }^{\forall} t \in\left[\tau_{k}, \tau_{k+1}\right) .
$$




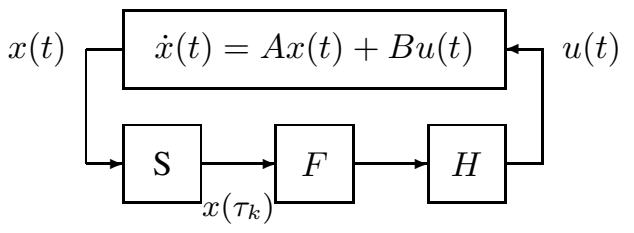

Fig. 1. Feedback control with aperiodic sample and hold actions

See also Fig. 1. Note that (3) implies

$$
\lim _{k \rightarrow \infty} \tau_{k}=\infty
$$

since $h_{\ell}>0$. Applications of this scenario can be found in networked and/or embedded control systems [2], [3], where resources for measurement and control are restricted.

The purpose of this paper is to provide stability criteria for $T$. If $\tau_{k}$ 's satisfy

$$
\tau_{k+1}-\tau_{k}=\tilde{h}
$$

for some $\tilde{h} \in\left[h_{\ell}, h_{u}\right]$, the resultant feedback control system is periodic. This special scenario is the one well-studied in the so-called sampled-data control theory [1]. Indeed the stability can be easily verified by checking the spectral radius of $\Phi(\tilde{h})$ in the special scenario, where

$$
\Phi(h):=\mathrm{e}^{A h}+\int_{0}^{h} \mathrm{e}^{A(h-\eta)} B \mathrm{~d} \eta F .
$$

It is, however, obvious that our general scenario is much more complicated, because of the uncertainly time-varying nature.

In this paper we will verify the stability of $T$ based on the following lemma [6], [3]:

Lemma 1: The origin of $T$ is exponentially stable if there exists a matrix $0<P=P^{*} \in \mathbb{R}^{n \times n}$ satisfying

$$
(\Phi(h))^{*} P \Phi(h)-P<0
$$

for all $h \in\left[h_{\ell}, h_{u}\right]$, where $\Phi(\cdot)$ is defined in (6).

Note that Lemma 1 is based on the quadratic stability of the accompanying discrete-time system $T_{d}$ defined by

$$
\xi[k+1]=\Phi\left(\tau_{k+1}-\tau_{k}\right) \xi[k]
$$

with the discrete-time Lyapunov function

$$
V(\xi[k]):=\xi^{*}[k] P \xi[k]
$$

where $\xi[k]:=x\left(\tau_{k}\right)$.

Note also that it is hard to find a matrix $P$ in Lemma 1 since the inequality must hold for all values in $\left[h_{\ell}, h_{u}\right]$, and $\Phi$ is a strongly nonlinear function of $h$.

Zhang-Branicky [6] proposes a randomized algorithm to search a $P$ on a grid between $h_{\ell}$ and $h_{u}$. In other words, the algorithm in [6] determines if a necessary condition for a sufficient condition for the stability holds or not, and hence cannot conclude the stability.

\section{Main Results}

The basic idea of the stability analysis in this paper is to imply the existence of $P>0$ satisfying (7) for all $h \in$ $\left[h_{\ell}, h_{u}\right]$ from that of $P>0$ satisfying (7) for all $h \in \mathcal{G}$ where $\mathcal{G}$ is a grid:

$$
\mathcal{G}=\left\{h_{1}, h_{2}, \ldots, h_{N}\right\} \subset\left[h_{\ell}, h_{u}\right] .
$$

Hence we discuss the robustness of systems with uniform sampling interval against the perturbation caused by the variation of sampling interval. It would be worth mentioning that most of existing results for stability of $T$ are derived by showing the robustness of continuous-time control system

$$
\dot{x}(t)=(A+B F) x(t)
$$

against the perturbation caused by the sampled-data action.

\section{A. Stability Criteria}

In order to discuss the robustness against the variation of sampling interval, we consider the following manipulation of $\Phi$ : Fix $h_{0} \in\left(h_{\ell}, h_{u}\right)$ and then one can define $\theta_{k}$ so that

$$
\tau_{k+1}-\tau_{k}=h_{0}+\theta_{k} .
$$

One has the following property, which is simple but plays a key role in this paper:

Proposition 1: The function $\Phi(\cdot)$ defined in (6) satisfies

$$
\Phi\left(\tau_{k+1}-\tau_{k}\right)=\Phi\left(h_{0}\right)+\Delta\left(\theta_{k}\right) \Psi\left(h_{0}\right)
$$

where

$$
\begin{aligned}
\Psi(h) & :=A \Phi(h)+B F, \\
\Delta(\theta) & :=\int_{0}^{\theta} \mathrm{e}^{A \eta} \mathrm{d} \eta .
\end{aligned}
$$

Proof: By definition

$$
\Phi\left(\tau_{k+1}-\tau_{k}\right)=\mathrm{e}^{A\left(h_{0}+\theta_{k}\right)}+\int_{0}^{h_{0}+\theta_{k}} \mathrm{e}^{A\left(h_{0}+\theta_{k}-\eta\right)} B \mathrm{~d} \eta F .
$$

The first term can be transformed to

$$
\mathrm{e}^{A\left(h_{0}+\theta_{k}\right)}=\mathrm{e}^{A \theta_{k}} \Phi\left(h_{0}\right)=\left(I+\Delta\left(\theta_{k}\right) A\right) \Phi\left(h_{0}\right) .
$$

While for the second term we have the following:

$$
\begin{aligned}
& \int_{0}^{h_{0}+\theta_{k}} \mathrm{e}^{A\left(h_{0}+\theta_{k}-\eta\right)} B \mathrm{~d} \eta \\
= & \int_{0}^{h_{0}} \mathrm{e}^{A\left(h_{0}+\theta_{k}-\eta\right)} B \mathrm{~d} \eta+\int_{h_{0}}^{h_{0}+\theta_{k}} \mathrm{e}^{A\left(h_{0}+\theta_{k}-\eta\right)} B \mathrm{~d} \eta \\
= & \mathrm{e}^{A \theta_{k}} \int_{0}^{h_{0}} \mathrm{e}^{A\left(h_{0}-\eta\right)} B \mathrm{~d} \eta+\Delta\left(\theta_{k}\right) B \\
= & \left(I+\Delta\left(\theta_{k}\right) A\right) \int_{0}^{h_{0}} \mathrm{e}^{A\left(h_{0}-\eta\right)} B \mathrm{~d} \eta+\Delta\left(\theta_{k}\right) B .
\end{aligned}
$$

Then it is straightforward to derive (8) by substituting the above results.

Now one can regard $T_{d}$ as a feedback connection of an LTI discrete-time system $\Sigma$ :

$$
\Sigma[z]:=\Psi\left(h_{0}\right)\left(z I-\Phi\left(h_{0}\right)\right)^{-1}
$$




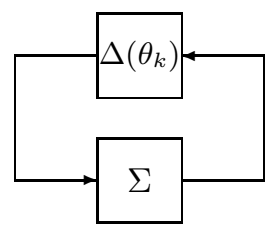

Fig. 2. Alternative representation of $T_{d}$

and a time-varying matrix $\Delta\left(\theta_{k}\right)$. See Fig. 2 . Thus we obtain the following lemma as a simple application of the smallgain theorem ${ }^{1}$ :

Lemma 2: Let an interval $\mathcal{H} \subseteq(0, \infty)$ be given. There exists a matrix $0<P=P^{*} \in \mathbb{R}^{n \times n}$ satisfying (7) for all $h \in \mathcal{H}$ if $\rho\left(\Phi\left(h_{0}\right)\right)<1$ and

$$
\gamma\|\Delta(\theta)\| \leq 1
$$

for all $\theta \in \mathcal{H}-h_{0}$, where $\gamma$ is an upper bound of $\|\Sigma\|_{\infty}$ :

$$
\gamma>\|\Sigma\|_{\infty}
$$

Since minimization of $\gamma$ in (11) is routine, one can verify the stability from (10) by bounding $\|\Delta(\theta)\|$. For the purpose we invoke the following property [18]:

Lemma 3: For given $A \in \mathbb{R}^{n \times n}$ and $t \geq 0$ one has

$$
\left\|\mathrm{e}^{A t}\right\| \leq \mathrm{e}^{\mu(A) t}
$$

where $\mu(A)$ denotes the $\log$ norm of $A$ :

$$
\mu(A)=\lambda_{\max }\left(\frac{A+A^{*}}{2}\right) .
$$

Remark 1: One can continue the following discussion by replacing the bound in (12) by other bounds found in, e.g., [18], [19].

In order to state the main results of this paper we need the following interval defined with given $h>0$ and $\gamma>0$ :

$$
\mathcal{H}(h, \gamma):=\left[h_{L}, h_{U}\right] \cap(0, \infty)
$$

where $h_{L}$ and $h_{U}$ are given as follows:

L1) If $\mu(-A)=0, h_{L}=h-\gamma^{-1}$,

L2) else if $\mu(-A) \leq-\gamma, h_{L}=-\infty$,

L3) else

$$
h_{L}=h-\frac{1}{\mu(-A)} \log \left(1+\gamma^{-1} \mu(-A)\right) .
$$

U1) If $\mu(A)=0, h_{U}=h+\gamma^{-1}$,

U2) else if $\mu(A) \leq-\gamma, h_{U}=\infty$,

U3) else

$$
h_{U}=h+\frac{1}{\mu(A)} \log \left(1+\gamma^{-1} \mu(A)\right) .
$$

Now we are ready to state the basic robustness results:

Theorem 1: Let $h_{0}>0$ be given so that $\rho\left(\Phi\left(h_{0}\right)\right)<1$. For $\gamma>0$ satisfying (11), there exists a matrix $0<P=$ $P^{*} \in \mathbb{R}^{n \times n}$ satisfying (7) for all $h \in \mathcal{H}\left(h_{0}, \gamma\right)$.

\section{Proof: See Appendix.}

\footnotetext{
${ }^{1}$ Readers are referred to, e.g., [17] on the relationship between the quadratic stability and the small-gain condition.
}

\section{B. Algorithm for Stability Analysis}

Theorem 1 provides a robustness condition for $T$ based on the property of the nominal system determined by the fixed sampling period $h_{0}$. A direct use of Theorem 1, however, can be conservative in the sense that there might not exist an $h_{0}>0$ such that $\left[h_{\ell}, h_{u}\right] \subseteq \mathcal{H}\left(h_{0}, \gamma\right)$ even though there exists a matrix $P$ satisfying (7) for all $h \in\left[h_{\ell}, h_{u}\right]$, mainly because of the small-gain type modeling of $\Delta\left(\theta_{k}\right)$.

In order to reduce the conservatism we introduce the multimodel to obtain the following theorem:

Theorem 2: Let $h_{i}>0(i=1,2, \ldots, N)$ be given. If there exist a matrix $0<X=X^{*} \in \mathbb{R}^{n \times n}$ and $\alpha_{i}>0$ $(i=1,2, \ldots, N)$ satisfying $N$ matrix inequalities

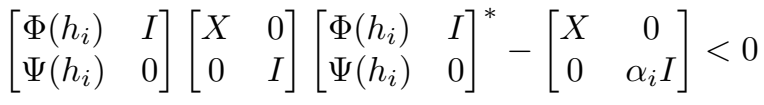

then (7) is satisfied with $P=X^{-1}$ for all

$$
h \in \bigcup_{i=1}^{N} \mathcal{H}\left(h_{i}, \sqrt{\alpha_{i}}\right)
$$

where $\Phi(\cdot), \Psi(\cdot), \mathcal{H}(\cdot, \cdot)$ are defined in (6), (9), and (13), respectively.

Proof: Consider the case $i=1$. The condition (14) with $i=1$ is an equivalent representation of

$$
\left\|\Psi\left(h_{1}\right)\left(z I-\Phi\left(h_{1}\right)\right)^{-1}\right\|_{\infty}<\sqrt{\alpha_{i}} .
$$

Hence, by invoking Theorem 1 , there exists a matrix $0<$ $P=P^{*} \in \mathbb{R}^{n \times n}$ satisfying (7) for all $h \in \mathcal{H}\left(h_{1}, \sqrt{\alpha_{1}}\right)$. Moreover we can verify that one of such $P$ is given by $X^{-1}$ from the standard procedure. With similar discussion, we can conclude that there exists a matrix $0<P=P^{*}=X^{-1} \in$ $\mathbb{R}^{n \times n}$ satisfying (7) for all $h \in \mathcal{H}\left(h_{i}, \sqrt{\alpha_{i}}\right), i=2, \ldots, N$. This concludes the proof.

Once we find a matrix $P>0$ satisfying (7) on a grid by any methods, e.g., one proposed in [6], we can verify the robustness by invoking Theorem 2 . In this paper we propose the following concrete algorithm for stability analysis which generates a grid effectively based on Theorem 2:

Algorithm 1: Given $0<h_{\ell}<h_{u}<\infty$, and a large positive integer $N_{0}$.

0. Initialization: $\mathcal{G} \leftarrow\left\{\left(h_{\ell}+h_{u}\right) / 2\right\}$

1. If there exists an $h \in \mathcal{G}$ satisfying $\rho(\Phi(h)) \geq 1$, the origin of $T$ is unstable. Stop.

2. If $\#(\mathcal{G}) \geq N_{0}$, stop without deciding the stability of the origin of $T$. Here $\#(\mathcal{G})$ denotes the number of elements in $\mathcal{G}$.

3. Minimize

$$
\sum_{i=1}^{\#(\mathcal{G})} \frac{\beta_{i}}{\left\|\Sigma_{i}\right\|_{\infty}^{2}}
$$

subject to

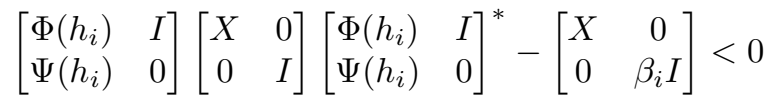

for all $h_{i}$ 's and $X>0$, where

$$
\Sigma_{i}[z]:=\Psi\left(h_{i}\right)\left(z I-\Phi\left(h_{i}\right)\right)^{-1},
$$


4. If

and $h_{i}$ is the $i$-th smallest element in $\mathcal{G}$.

$$
\left[h_{\ell}, h_{u}\right] \subseteq \bigcup_{i=1}^{\#(\mathcal{G})} \mathcal{H}\left(h_{i}, \sqrt{\alpha_{i}}\right),
$$

the origin of $T$ is exponentially stable. Stop. Here

$$
\alpha_{i}:=\lambda_{\max }\left(R_{i}-S_{i}^{*}\left(Q_{i}-X_{i}\right)^{-1} S_{i}\right)+\varepsilon
$$

where $\varepsilon$ is a small positive number and

$$
\left[\begin{array}{ll}
Q_{i} & S_{i} \\
S_{i}^{*} & R_{i}
\end{array}\right]:=\left[\begin{array}{ll}
\Phi\left(h_{i}\right) & I \\
\Psi\left(h_{i}\right) & 0
\end{array}\right]\left[\begin{array}{cc}
X & 0 \\
0 & I
\end{array}\right]\left[\begin{array}{lll}
\Phi\left(h_{i}\right) & I \\
\Psi\left(h_{i}\right) & 0
\end{array}\right]^{*} .
$$

5. Update $\mathcal{G}$ by

$$
\mathcal{G} \leftarrow \mathcal{G} \cup\left\{\left(L_{j}+U_{j}\right) / 2\right\}
$$

for all $j$ where $L_{j}$ and $U_{j}$ are determined so that

$$
\begin{gathered}
\bigoplus_{j=1}^{M}\left(L_{j}, U_{j}\right)=\left(h_{\ell}, h_{u}\right) \backslash\left(\left(h_{\ell}, h_{u}\right) \cap \bigcup_{i=1}^{\#(\mathcal{G})} \mathcal{H}\left(h_{i}, \sqrt{\alpha_{i}}\right)\right), \\
L_{1}<U_{1}<L_{2}<U_{2}<\cdots<L_{M}<U_{M}
\end{gathered}
$$

are satisfied. Go to Step 1.

We have some remarks for Algorithm 1: Step 2 is introduced to avoid numerical issues which could happen when $\#(\mathcal{G})$ is too large. The performance of the algorithm can be tuned by modifying the objective function in Step 3 . Note that $\alpha_{i}$ satisfies (14) with $X$ determined in Step 3 and $\alpha_{i} \leq \beta_{i}$ with sufficiently small $\varepsilon$. The integer $M$ in Step 4 is $\#(\mathcal{G})+1$ at most.

\section{NUMERICAL EXAMPLES}

In this section we demonstrate the validity of the proposed method for stability analysis.

\section{A. Example in [6]}

Let us consider the following problem parameters [6]:

$$
A=\left[\begin{array}{cc}
0 & 1 \\
0 & -0.1
\end{array}\right], \quad B=\left[\begin{array}{c}
0 \\
0.1
\end{array}\right], \quad F=-\left[\begin{array}{ll}
3.75 & 11.5
\end{array}\right] .
$$

We search for an interval $\tilde{\mathcal{H}}$ satisfying that the origin of $T$ is exponentially stable if

$$
\tau_{k+1}-\tau_{k} \in \tilde{\mathcal{H}}
$$

Applying the methods in the literature we obtain several $\tilde{\mathcal{H}}$ 's: From the results in [4] we have $\left(0,2.7 \times 10^{-4}\right.$ ] (reported in [5]). It is improved to $\left(0,4.5 \times 10^{-4}\right]$ in [5], and $(0,0.0593]$ in [6]. The input delay approach significantly improved the conservatism to have $(0,0.869$ ] in [7], [8], [10]. One can find further improvements: $(0,0.887]$ in [11], $(0,1.113]$ in [13], [14], and $(0,1.365]$ in [15].

It would be natural to take $h_{\ell}>0$ for practical situations of networked/embedded control systems. There are less results which explicitly consider the case of $h_{\ell}>0$. We obtain $[0.01,0.87]$ from the results in [10] but this is included in $(0,1.365]$ in [15].
In contrast to the existing results, the proposed algorithm proved the exponential stability of the origin of $T$ for $\tilde{\mathcal{H}}=$ $[0.01,1.72]$ with

$$
P=\left[\begin{array}{cc}
4.03 & 5.09 \\
5.09 & 13.49
\end{array}\right] \times 10^{-3} .
$$

We remark that $T$ is unstable when the sampling period is fixed to 1.73 .

We have implemented Algorithm 1 on MATLAB 7.4, YALMIP (R20070523) [20], and SDPT3 (4.0 $\beta$ ) [21]. The search took 6.33 [s] on a laptop with Intel Core 2 Duo $(2.33 \mathrm{GHz})$ running MacOSX, and the maximal $\#(\mathcal{G})$ in the search was 25 .

\section{B. Example in [16]}

Let us consider the following problem parameters [16]:

$$
A=-2, \quad B=F=1 .
$$

The stability of $T$ is verified for any fixed $h_{u}<\infty$ in [16], where the passivity theorem plays the key role. Note that most of other existing results cannot conclude the stability for large $h_{u}$. Now let us apply the proposed methods in this paper: One can verify that

$$
\left\|\Psi\left(h_{0}\right)\left(z I-\Phi\left(h_{0}\right)\right)^{-1}\right\|_{\infty}=\frac{2 \mathrm{e}^{-2 h_{0}}}{\mathrm{e}^{-2 h_{0}}+3}<\frac{1}{4}<2
$$

and hence Theorem 1 guarantees the stability for any large $h_{u}$ (Case U2 in the definition of $\mathcal{H}$ ).

It seems to be unlikely that one can guarantee the stability for large $h_{u}$ with small-gain type discussion in the continuous-time domain if we take a time-invariant system as the nominal part, since the perturbation can grow extremely large for large $h_{u}$. Hence this example clarifies one of the benefits to take the discrete-time approach, noting that we have taken the small gain approach in the discrete-time domain.

\section{EXTENSIONS FOR CONSERVATISM REDUCTION}

The proposed algorithm chops the given interval $\left[h_{\ell}, h_{u}\right]$ into pieces to verify the stability by using Theorem 1 . Although it enables to test the stability for large range of the sampling interval in spite of the conservatism in Theorem 1, it is obvious that the performance of the algorithm is improved if one can reduce the conservatism in Theorem 1. There are several directions for the purpose. In this section we suggest and discuss some of them with numerical evaluation.

A straightforward way is to replace the bound of the maximal singular value of matrix exponential in (12) by other bounds found in, e.g., [18], [19]. Since the performance of the bound depends on the matrix taken the exponential [18], [19], which is the ' $A$ '-matrix of the plant in our problem, it might be practical to use bounds as many as possible if computational time is allowed.

Another way to reduce the conservatism is to replace the small gain condition (10) by a general quadratic condition 


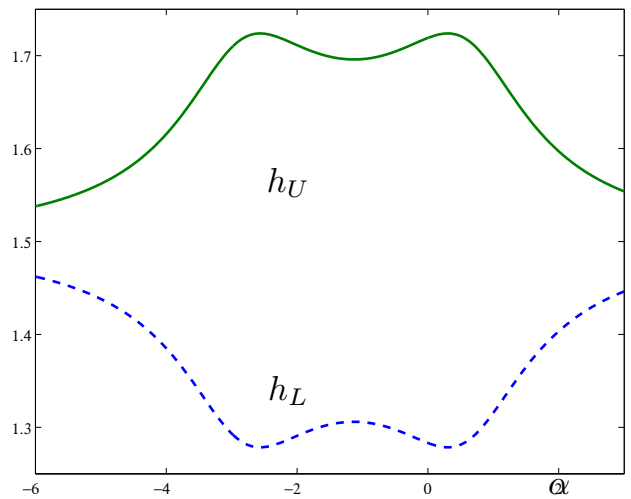

Fig. 3. $h_{U}$ and $h_{L}$ varying $\alpha$

in, e.g., [22], [23], [24]. For the generalization it is required to find a matrix $\Pi=\Pi^{*} \in \mathbb{R}^{(n+m) \times(n+m)}$ satisfying

$$
\left[\begin{array}{l}
I \\
\Delta
\end{array}\right]^{*} \Pi\left[\begin{array}{l}
I \\
\Delta
\end{array}\right] \geq 0
$$

for all $\Delta \in\left\{\Delta(\theta), \theta \in\left[h_{\ell}-h_{0}, h_{u}-h_{0}\right]\right\}$. One such $\Pi$ is given by

$$
\Pi=\left[\begin{array}{cc}
\mathrm{e}^{-A^{*} \alpha} \mathrm{e}^{-A \alpha} & 0 \\
0 & -\gamma^{2} \mathrm{e}^{-A^{*} \alpha} \mathrm{e}^{-A \alpha}
\end{array}\right]
$$

for all $\alpha \in \mathbb{R}$ and $\gamma \leq 1 /\|\Delta\|$, noting that $\Delta(\theta)$ and $\mathrm{e}^{A \alpha}$ commute. Note that this $\Pi$ is related to the scaled small gain condition and one can reduce the conservatism in Theorem 1 by replacing $\|\Sigma\|_{\infty}$ by $\left\|\mathrm{e}^{-A \alpha} \Sigma \mathrm{e}^{A \alpha}\right\|_{\infty}$. For the problem data in Section IV-A with $h_{0}=0.1$, Theorem 1 and the above condition verify the stability for $h_{0}+[-6.85,6.87] \times 10^{-2}$ and $h_{0}+[-9.52,9.57] \times 10^{-2}$ respectively, where we swept $\alpha$ from -6 to 3 with 0.01 step. This is more than $35 \%$ improvement. One drawback of this method is the fact that the corresponding optimization problem is not convex: For the problem data in Section IV-A with $h_{0}=1.5$, we plot

$$
\left[h_{L}, h_{U}\right]=\mathcal{H}\left(h_{0}, \gamma\right)
$$

for $\gamma>\left\|\mathrm{e}^{-A \alpha} \Sigma \mathrm{e}^{A \alpha}\right\|_{\infty}$ in Fig. 3, varying $\alpha$. We see that both $h_{L}$ and $h_{U}$ are multimodal in $\alpha$.

Finally let us notice that the conservatism is Theorem 1 is more serious for very small $h_{0}$ since $\Phi\left(h_{0}\right)$ is close to the identity and $\gamma$ is large. One might reduce the conservatism by modifying the modeling. Noting that

$$
\Delta(\theta)=\int_{0}^{\theta}\left(\mathrm{e}^{A t}-I+I\right) \mathrm{d} t=\theta I+\int_{0}^{\theta}\left(\mathrm{e}^{A t}-I\right) \mathrm{d} t,
$$

one can show that the exponential stability of origin of $T$ for $h \in[-\theta, \theta]+h_{0}$ if there exist a matrix $P>0$ and $\alpha$ satisfying

$$
\begin{aligned}
& {\left[\begin{array}{cc}
\Omega_{+}\left(h_{0}, \theta\right) & I \\
\Psi\left(h_{0}\right) & 0
\end{array}\right]\left[\begin{array}{cc}
X & 0 \\
0 & I
\end{array}\right]\left[\begin{array}{cc}
\Omega_{+}\left(h_{0}, \theta\right) & I \\
\Psi\left(h_{0}\right) & 0
\end{array}\right]^{*}-\left[\begin{array}{cc}
X & 0 \\
0 & \alpha I
\end{array}\right]<0,} \\
& {\left[\begin{array}{cc}
\Omega_{-}\left(h_{0}, \theta\right) & I \\
\Psi\left(h_{0}\right) & 0
\end{array}\right]\left[\begin{array}{cc}
X & 0 \\
0 & I
\end{array}\right]\left[\begin{array}{cc}
\Omega_{-}\left(h_{0}, \theta\right) & I \\
\Psi\left(h_{0}\right) & 0
\end{array}\right]^{*}-\left[\begin{array}{cc}
X & 0 \\
0 & \alpha I
\end{array}\right]<0,}
\end{aligned}
$$

$$
\frac{\sqrt{\alpha}}{2} \frac{\|A\|}{1+\|A\|} \theta^{2} \mathrm{e}^{\|A\| \theta} \leq 1,
$$

where

$$
\Omega_{ \pm}\left(h_{0}, \theta\right):=\Phi\left(h_{0}\right) \pm \theta \Psi\left(h_{0}\right) .
$$

In the derivation the following property [18] plays the key role:

$$
\left\|\mathrm{e}^{A t}-I\right\| \leq t\|A\| \mathrm{e}^{\|A\| t},
$$

although the detail is omitted. For the problem data in Section IV with $h_{0}=0.01$, Theorem 1 and the above condition verify the stability for $h_{0}+[-6.79,6.79] \times 10^{-4}$ and $h_{0}+[-9.50,9.50] \times 10^{-4}$ respectively (more than $40 \%$ improvement is achieved). One drawback of this method is the dependence of conditions to be checked on $\theta$ which makes the algorithm more complicated.

\section{CONCLUding REMARKS}

We have considered the stability of sampled-data feedback control systems where the state is sampled aperiodically, motivated by widespread use of networked and embedded control systems.

We have proposed a stability analysis algorithm by showing robustness of sampled-data systems against perturbation caused by variation of sampling intervals based on the smallgain framework. We have also discussed some directions for reducing the conservatism.

In this paper we have considered an analysis problem for a simple sampled state feedback scenario, however, application to more practical analysis and synthesis problems are not hard.

\section{ACKNOWLEDGMENTS}

The author would like to express his appreciations to Prof. Mirkin who introduced the author to this subject with fruitful discussions.

\section{REFERENCES}

[1] T. Chen and B. Francis, Optimal Sampled-Data Control Systems. Springer, 1995.

[2] D. Hristu-Varsakelis and W. Levine, eds., Handbook of Networked and Embedded Control Systems. Birkhäuser, 2005.

[3] J. Hespanha, P. Naghshtabrizi, and Y. Xu, "A survey of recent results in networked control systems," Proceedings of the IEEE, vol. 95, pp. 138-162, 2007.

[4] G. Walsh, H. Ye, and L. Bushnell, "Stability analysis of networked control systems," in Proc. American Control Conf., pp. 2876-2880, 1999.

[5] W. Zhang, M. Branicky, and S. Phillips, "Stability of networked control systems," IEEE Control Systems Maganine, vol. 21, pp. 84-99, 2001.

[6] W. Zhang and M. Branicky, "Stability of networked control systems with time-varying transmission period," in Allerton Conf. Communication, Contr. and Computing, 2001.

[7] E. Fridman, A. Seuret, and J. Richard, "Robust sampled-data stabilization of linear systems: an input delay approach," Automatica, vol. 40, pp. 1441-1446, 2004.

[8] D. Yue, Q. Han, and C. Peng, "State feedback controller design of networked control systems," IEEE Transactions on Circuits and Systems II, vol. 51, pp. 640-644, 2004.

[9] E. Fridman, U. Shaked, and V. Suplin, "Input/output delay approach to robust sampled-data $H_{\infty}$ control," Systems \& Control Letters, vol. 54, pp. 271-282, 2005. 
[10] P. Naghshtabrizi and J. Hespanha, "Designing an observer-based controller for a network control system," in Proceedings of the 44th IEEE Conference on Decision and Control, and the European Control Conference 2005, pp. 848-853, 2005.

[11] D. Yue, Q. Han, and J. Lam, "Network-based robust $H_{\infty}$ control of systems with uncertainty," Automatica, vol. 41, pp. 999-1007, 2005.

[12] V. Suplin, E. Fridman, and U. Shaked, "Sampled-data $H_{\infty}$ control and filtering: Nonuniform uncertain sampling," Automatica, vol. 43, pp. 1072-1083, 2007.

[13] P. Naghshtabrizi, J. Hespanha, and R. Teel, "On the robust stability and stabilization of sampled-data systems: A hybrid system aproach," in Proceedings of the 45th IEEE Conference on Decision and Control, pp. 4873-4878, 2006.

[14] P. Naghshtabrizi, J. Hespanha, and A. Teel, "Stability of delay impulsive systems with application to networked control systems," in Proc. American Control Conf., 2007.

[15] L. Mirkin, "Some remarks on the use of time-varying delay to model sample-and-hold circuits," IEEE Transactions on Automatic Control, vol. 52, pp. 1109-1112, 2007.

[16] H. Fujioka, "Stability analysis of systems with aperiodic sample-andhold devices," in Proceedings of the IFAC 7th Workshop on Time-Delay Systems, 2007. to appear.

[17] P. Khargonekar, I. Petersen, and K. Zhou, "Robust stabilization of uncertain linear systems: Quadratic stabilizability and $H^{\infty}$ control theory," IEEE Transactions on Automatic Control, vol. 35, pp. 356361, 1990.

[18] C. V. Loan, "The sensitivity of the matrix exponential," SIAM Journal of Numerical Analysis, vol. 14, pp. 971-981, 1977.

[19] B. Kågström, "Bounds and perturbation bounds for the matrix exponential," BIT, vol. 17, pp. 89-97, 1977.

[20] J. Löfberg, "YALMIP : A toolbox for modeling and optimization in MATLAB," in Proceedings of the IEEE CACSD Conference, 2004.

[21] R. Tutuncu, K. Toh, and M. Todd, "Solving semidefinite-quadraticlinear programs using SDPT3," Mathematical Programming Ser. B, vol. 95, pp. 189-217, 2003.

[22] A. Megretski and A. Rantzer, "System analysis via integral quadratic constraints," IEEE Transactions on Automatic Control, vol. 47, pp. 819-830, 1997.

[23] T. Iwasaki and S. Hara, "Well-posedness of feedback systems: Insights into exact robustness analysis and approximate computations," IEEE Transactions on Automatic Control, vol. 48, pp. 619-630, 1998.

[24] C. Scherer, "Robust mixed control and LPV control with full block scalings," in Recent Advances on LMI Methods in Control (S. N. L. El Ghaoui, ed.), SIAM, 2000

\section{APPENDIX}

\section{Proof of Theorem 1}

We here prove that (10) holds for all $h \in\left[h_{0}, h_{U}\right]$. The proof for the interval $\left[h_{L}, h_{0}\right]$ is similar so it is omitted. Note that $H\left(h_{0}, \gamma\right) \subseteq\left[h_{L}, h_{U}\right]$.

Invoking Lemma 3 we have

$$
\|\Delta(\theta)\| \leq \int_{0}^{\theta}\left\|\mathrm{e}^{A t}\right\| \mathrm{d} t \leq \int_{0}^{\theta} \mathrm{e}^{\mu(A) t} \mathrm{~d} t
$$

when $\theta \geq 0$. If $\mu(A)=0$

$$
\|\Delta(\theta)\| \leq \theta .
$$

Hence (10) holds as long as $\gamma \theta \leq 1$. This completes the proof for the case $\mathrm{U} 1$.

Let us next consider the case of $\mu(A) \neq 0$. In this case

$$
\|\Delta(\theta)\| \leq \frac{\mathrm{e}^{\mu(A) \theta}-1}{\mu(A)} .
$$

Suppose that $\mu(A)<0$. Noting that the right hand side goes to $-1 / \mu(A)$ when $\theta$ tends to $\infty$. Hence (10) holds for all $\theta>0$ if

$$
-\frac{\gamma}{\mu(A)} \leq 1
$$

This completes the proof for the case U2.

Finally let us consider the case of $\mu(A) \neq 0$ and

$$
-\frac{\gamma}{\mu(A)}>1
$$

The small gain condition (10) holds for all $\theta>0$ if

$$
\gamma \frac{\mathrm{e}^{\mu(A) \theta}-1}{\mu(A)} \leq 1
$$

Noting that $1+\gamma^{-1} \mu(A)>0$ in this case, this condition turns to

$$
\begin{aligned}
& \text { Case A) If } \mu(A)>0 \\
& \qquad \mu(A) \theta \leq \log \left(1+\gamma^{-1} \mu(A)\right) .
\end{aligned}
$$

Case B) If $\mu(A)<0$

$$
\mu(A) \theta \geq \log \left(1+\gamma^{-1} \mu(A)\right) .
$$

Hence we have

$$
\theta \geq \frac{1}{\mu(A)} \log \left(1+\gamma^{-1} \mu(A)\right)
$$

for both cases. This completes the proof for the case U3. 